\title{
Las intervenciones ambientales para la conservación de bosques comunitarios en la Comunidad Nativa Siete de junio del distrito de Palcazú, Oxapampa, Pasco (Perú)
}

\author{
Environmental interventions for the conservation of community forests in the \\ Native Community of Siete de junio of the Palcazu district, Oxapampa, Pasco
} (Peru)

Alex G. Avendaño ${ }^{1, *}$

Recibido: 19 agosto 2020 | Aceptado: 28 setiembre 2020 | Publicado en línea: 12 diciembre 2020 Citación: Avendaño, AG. 2020. Las intervenciones ambientales para la conservación de bosques comunitarios en la Comunidad Nativa Siete de junio, Palcazú, Perú. Revista Forestal del Perú 35 (3, Número Especial): 28-43. DOI: http://dx.doi.org/10.21704/rfp.v35i3.1599

\begin{abstract}
Resumen
Este artículo trata acerca de los esfuerzos para la conservación ambiental y el manejo de los recursos en las tierras de la Comunidad Nativa de Siete de junio del valle del Palcazú (provincia de Oxapampa, región Pasco), cuyos habitantes fueron los actores centrales de un trabajo de campo realizado entre los meses de abril y mayo del 2019. Con base en las fuentes de información primaria y secundaria, se describen los cambios y continuidades en los usos de los suelos de la comunidad, así como la relación de los yánesha con los proyectos ambientales de los últimos cinco años. Se hallaron cambios en el uso del suelo a raíz del aumento de la superficie agrícola, interpretándose en relación con los proyectos de desarrollo sostenible basados en el cultivo del cacao; y se constató el papel que las nuevas formas institucionalizadas de organización entre los yánesha juegan en estos proyectos. De esta manera, se argumenta que las intervenciones para la conservación de los bosques comunitarios del Palcazú se han aproximado a sus objetivos al incorporar las intervenciones para desarrollo sostenible y las nuevas formas organizativas. No obstante, también se sostiene que el desarrollo de estos programas y proyectos deben matizarse con los contextos socioambientales, sutilmente variables, de las comunidades del Palcazú. Entre ellas, la C.N. Siete de junio permite reflexionar sobre los alcances y limitaciones de las intervenciones para la conservación de los bosques comunitarios.
\end{abstract}

Palabras clave: bosque comunitario, conservación, desarrollo sostenible, pueblos indígenas, yánesha

${ }^{1}$ Grupo de Antropología Amazónica - PUCP

* Autor de Correspondencia: alexg.avendano@pucp.pe 


\begin{abstract}
This article deals with the course of the efforts for the environmental conservation and forest resource management in the lands belonging to Comunidad Nativa Siete de junio of the Palcazu valley (Oxapampa province, Pasco region), whose inhabitants were the central actors of a fieldwork carried out between April and May 2019. Taking into account primary and secondary sources of information, the changes and continuities in the community's land uses and the relationship of the yanesha people with the environmental projects, within the last five years, are described. Changes in land use were found as a result of the increase in agricultural surface; and the role that the new institutionalized forms of organization among the yanesha play in these projects was verified. Thus, I argue that the projects for the conservation of Palcazu's communities' forests have approached their goals by incorporating the interventions for sustainable development and the new institutionalized forms. However, I also contend that the development of these projects and programs must be considered against the subtly variable socio-environmental contexts of the communities of the Palcazú. Among them, the C.N. Siete de junio allows to reflect upon the scopes and limitations of the communal forests' conservation interventions.
\end{abstract}

Key words: community forest, conservation, indigenous peoples, sustainable development, yanesha

\section{Introducción}

Las preocupaciones actuales en torno a la conservación ambiental de la Amazonía peruana se asientan sobre una historia de discursos y prácticas cambiantes sobre el curso de su desarrollo idóneo. En el valle del río Palcazú, ubicado en el distrito homónimo de la provincia de Oxapampa, en la región Pasco, esta historia se ha desenvuelto a partir de la segunda mitad del siglo veinte. Desde inicios de la década de 1980, sus bosques y habitantes han sido, respectivamente, objetos y sujetos de un conjunto de intervenciones con objetivos explícitos de conservación y desarrollo sostenible (Smith 1982; Hartshorn y Stocks 1993; Staver et al. 1994; Larsen 2016). Entre estas iniciativas, resaltan los programas y proyectos cuyos beneficiarios y participantes son los miembros del pueblo yánesha, quienes viven en las comunidades nativas (en adelante CC.NN.) del valle e interactúan con la Reserva Comunal Yanesha (RCY), un área natural protegida (en adelante ANP) establecida en 1988.

Los yánesha son un pueblo indígena cuya lengua pertenece a la familia lingüística Arawak (Santos-Granero 2004). Con un número aproximado de 9,400 miembros hacia el año 2017, los yánesha se encuentran viviendo alrededor de la cuenca del río Palcazú, la margen izquierda del Alto Perené, y la cuenca del Alto Pachitea; $y$, en menor medida, en el valle de Oxapampa (Santos-Granero 2004; INEI 2018). Una serie de investigaciones desde la antropología y la etnobotánica se han aproximado cuantitativa y cualitativamente a las actividades productivas y extractivas que los yánesha han empleado para su sustento local y el comercio, y que han transformado los bosques del Palcazú en décadas pasadas (Smith 1982; Salick 1989; Staver 1989; Hartshorn y Salick 2003; Santos-Granero 2004). En la actualidad, se evidencia la predominancia de los proyectos de desarrollo sostenible basados en la inserción de ciertas actividades comerciales, como la piscicultura y el cultivo comercial de cacao, entre los habitantes yánesha de las CC.NN. del Palcazú (Luján Berger 2017); pero surgen algunas interrogantes sobre otras actividades que buscan impedir y/o revertir la pérdida de bosques de las CC.NN. y la RCY.

Este artículo parte de la pregunta de cómo describir el desarrollo en el tiempo de las intervenciones dirigidas a la conservación de los bosques situados dentro de las CC.NN. del curso medio del río Palcazú. El objetivo de este estudio consiste en describir e interpretar los cambios en el uso de los suelos en la C.N. Siete de junio con relación a los programas y proyectos para conservación y desarrollo sostenibles, 

en la Comunidad Nativa Siete de junio, Perú

realizados con sus comuneros desde el año 2015. Así, se argumentará que las intervenciones ambientales para la conservación de los bosques comunitarios ${ }^{1}$ del Palcazú se han aproximado a su objetivo en la medida en que su diseño ha incorporado las intervenciones para el desarrollo sostenible en marcha y formas institucionalizadas de organización relativamente nuevas entre los habitantes yánesha. Sin embargo, los programas y proyectos para la conservación de los bosques comunitarios se asientan en contextos socioambientales e históricos que varían sutilmente entre las comunidades del valle. Estos, a su vez, hacen que los intentos de implantar prácticas de conservación y manejo de recursos entre los comuneros yánesha lleven a diferentes resultados.

El argumento descrito se expondrá en las siguientes secciones. En primer lugar, se establecerá la metodología establecida para responder la pregunta de este estudio. Esta se basa en la combinación de información cualitativa, recogida mediante un trabajo de campo con enfoque etnográfico, e información cuantitativa, proveniente de fuentes secundarias. En segundo lugar, se describirá la historia de los cambios en el uso de las tierras en las CC.NN. del Medio Palcazú y la C.N. Siete de junio. Luego, se describirán e interpretarán las actividades de la ONG Desarrollo Rural Sustentable (DRIS) como intervenciones ambientales efectuadas sobre los comuneros del sector Pampacocha de la C.N. Siete de junio. Finalmente, se establecerá una discusión breve sobre los proyectos de conservación y desarrollo sostenible contemporáneos entre los pueblos indígenas y se trazaran las conclusiones. Estas últimas incluirán algunas recomendaciones, basadas en los resultados del estudio, para futuras intervenciones de carácter ambiental.

\section{Materiales y Métodos}

En este estudio, se adoptó un enfoque histórico y etnográfico para describir e interpretar las transformaciones en los bosques de la C.N. Siete de junio (Figura 1 y Figura 2), llevadas a cabo por sus habitantes yánesha desde la década de 1960. En aquella época comenzaron los cambios más significativos, debido al incremento de la población y a la expansión de las actividades ganaderas dentro de las comunidades (Smith 1982; Santos-Granero y Barclay 1995). La historia de cambios en el uso de la tierra en esta comunidad y el distrito de Palcazú se complementa con la información obtenida acerca del desarrollo de los programas y proyectos para la conservación de los bosques comunitarios en los últimos cinco años. Como se detallará más adelante, estos datos provienen de las entrevistas realizadas a diferentes actores asociados a estas intervenciones.

Para propósitos de interpretación de la información, se conciben a los programas y proyectos para conservación y desarrollo sostenible como intervenciones ambientales. Alineándose en las definiciones y debates de diferentes autores sobre los conceptos de environmentality y ecogubernamentalidad (Ulloa 2004; Agrawal 2005; Cepek 2011), las intervenciones ambientales se definen en este estudio como aquellas que buscan encauzar el comportamiento y la acción de los sujetos hacia fines ambientales. Estas pueden subdividirse en las categorías de intervenciones para conservación e intervenciones para desarrollo sostenible. Si bien las primeras son el objeto del estudio, su estrecho entrelazamiento con las segundas permite responder la pregunta planteada.

Esta investigación se basa en una metodología que combinó técnicas de recojo de información cualitativa y el empleo de información cuantitativa de fuentes secundarias. Las primeras fueron recogidas por el investigador mediante un método de trabajo de campo con enfoque etnográfico, realizado entre inicios de abril y finales de mayo del 2019. Este supuso una estadía entre los comuneros del sector Pampacocha de la C.N. Siete de junio, así como la

\footnotetext{
${ }^{1}$ En el contexto de este artículo, se emplea el término 'bosque comunitario' para referirse a la superficie de bosque abarcada dentro de la Comunidad Nativa; y no implica necesariamente un uso institucionalizado por parte de la población indígena.
} 


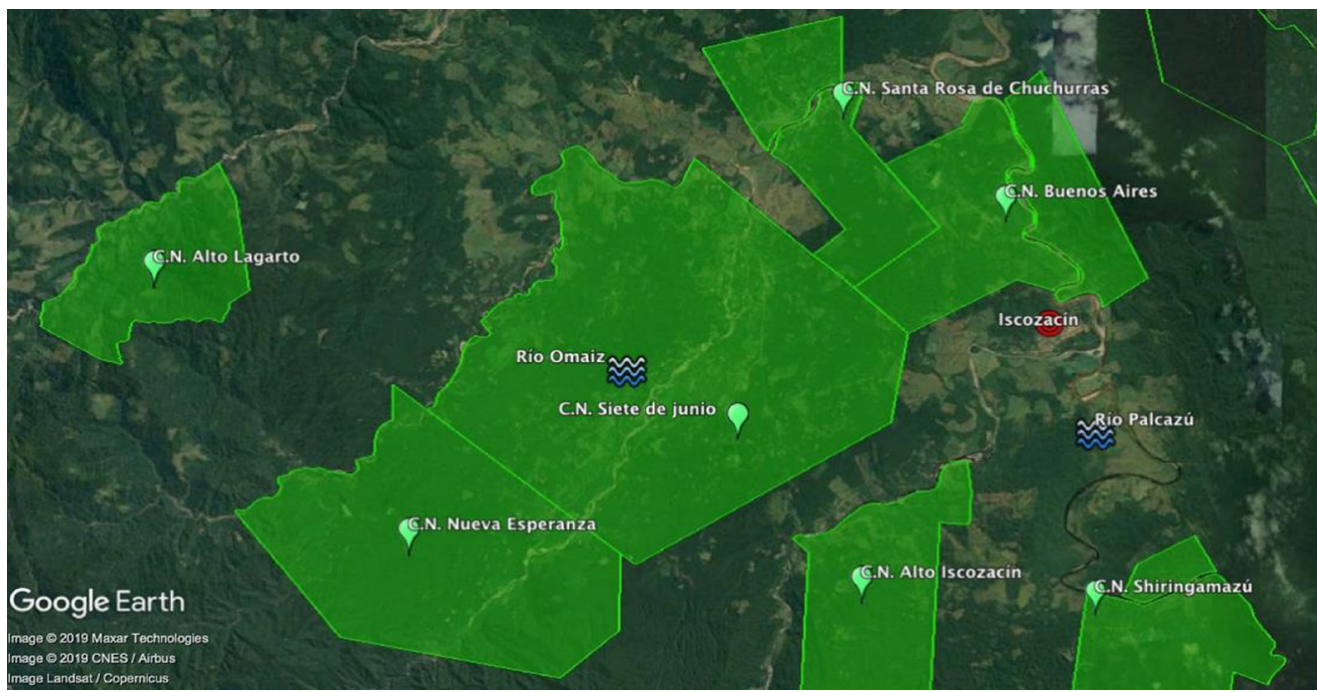

Figura 1. Mapa de la C.N. Siete de junio. Elaboración del autor en la plataforma Google Earth, con base en IBC (2020).

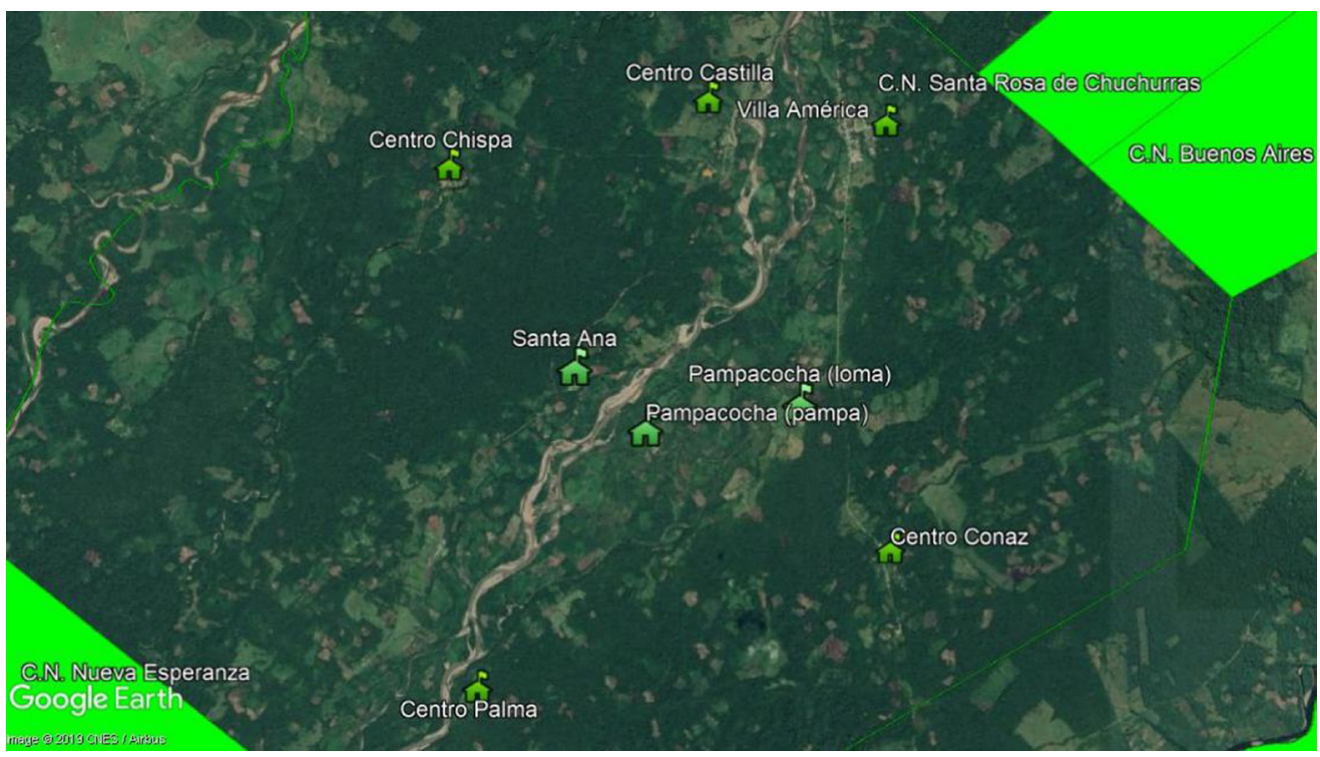

Figura 2. Mapa de los sectores visitados en el trabajo de campo. Elaboración del autor en la plataforma Google Earth, con base en IBC (2020).

apertura a otras dimensiones de la vida social vinculadas al tema de estudio. Dentro de este marco de convivencia, se recogió información adicional mediante conversaciones informales y entrevistas a profundidad con distintos actores vinculados a las intervenciones ambien- tales en marcha. En este estudio, se destacan la información obtenida con el jefe zonal de la ONG DRIS y dos comuneros [un promotor de los proyectos de cacao y un antiguo presidente de Asociación para el Manejo y la Conservación de la Reserva Comunal Yanesha 

en la Comunidad Nativa Siete de junio, Perú

(AMARCY) $]^{2}$ asociados de forma estrecha a las intervenciones ambientales pasadas en la comunidad y el sector de Pampacocha.

Mientras tanto, la información cuantitativa citada proviene de fuentes secundarias oficiales y estudios previos. Específicamente, la información empleada para describir los cambios en el uso de la tierra fue organizada a partir de los datos sobre capacidad de uso mayor del suelo en las CC.NN. del Palcazú (Smith 1982), los datos censales provenientes del Censo de Comunidades Nativas del IBC del 2000 (IBC 2006) y el II y III Censo de Comunidades Indígenas (INEI 2008, 2018), y los datos sobre cambios en el uso de la tierra del distrito de Palcazú disponibles en la plataforma web Geobosques (2019).

\section{Resultados}

\section{Una historia de cambios en el uso de la tierra}

El curso de los proyectos para conservación y desarrollo en el valle del Palcazú se asienta sobre una historia de cambios respecto al uso y tenencia de sus tierras. El río Palcazú, cuyo sentido va de sur a norte, forma una cuenca que se encuentra delimitada por la cordillera de Yanachaga, al oeste, y la cadena de San Matías, al este. El suelo del valle, por su parte, se extiende a lo largo del río Palcazú y sus afluentes (como los ríos Iscozacín y Chuchurras); pero resulta ser angosto (especialmente en su curso alto) debido al terreno escarpado que forman las cadenas montañosas ya mencionadas (Salick 1989; Hartshorn y Stocks 1993). En el curso medio del río Palcazú, donde se ubica la C.N. Siete de junio, comienza a aumentar la superficie conformada por el suelo del valle.

Desde finales del siglo XIX, el valle del Palcazú comenzó a albergar a una cantidad mayor de población yánesha a medida que estos se veían forzados a migrar debido a las presiones colonizadoras en su territorio original (Santos-Granero y Barclay 1995). No obstante, el ciclo extractivo del caucho contribuyó a que el Palcazú entrase en la órbita de los procesos económicos generalizados en la Amazonía de aquel entonces. Así, un conjunto de empresas caucheras reclamaron concesiones para la extracción de caucho en diferentes espacios del valle a inicios del siglo pasado (Santos-Granero y Barclay 1995). De igual manera, algunas familias de origen alemán establecieron haciendas ganaderas en las tierras más fértiles del valle.

Los hacendados, conocidos localmente como patrones, fueron apropiándose de mayores extensiones del valle durante las primeras décadas del siglo XX (Santos-Granero y Barclay 1995). Para poder abrir y emplear estas tierras, los hacendados requerían emplear la fuerza de trabajo de las familias yánesha que habitaban en asentamientos dispersos. La búsqueda por el consumo de bienes externos a la economía de subsistencia permitió la apertura de los hombres yánesha a trabajar en la apertura de pastizales para la crianza de ganado vacuno (Smith 1982). Por otro lado, los patrones monopolizaron la conexión entre los yánesha y la economía de mercado, empleando el sistema de habilitación y enganche para controlar la fuerza de trabajo de aquellos (Santos-Granero y Barclay 1995). Sobre la base de esta relación social, la transformación de los bosques del valle a pastizales no solo sería exclusiva a las tierras de los patrones.

La vinculación entre patrones y peones mediante el sistema de habilitación y enganche cimentó el desarrollo de una modalidad de crianza de ganado, denominada como el sistema de crianza al partir (Smith 1982; Santos-Granero y Barclay 1995). A través de este arreglo, el hacendado prestaba una cantidad de cabezas de ganado al hombre yánesha para que pastara en las tierras de este último. Tras el nacimiento de las crías, el hacendado solía reclamar la mitad de estas y el ganado original. El sistema de crianza al partir representó la cúspide de la dominación económica de los patrones ganaderos sobre los yánesha. En la década de

${ }^{2}$ En este estudio se ha elegido mantener el anonimato de los informantes. Sin embargo, se consigna su ocupación actual o cargo pasado. 
1970, Richard C. Smith (1982) constató que el 90\% de los hombres yánesha que residían en los asentamientos del curso medio del río Palcazú se encontraban bajo arreglos de crianza al partir con algún patrón. Esta modalidad de crianza tuvo dos grandes consecuencias para los yánesha del Medio Palcazú: por un lado, les permitió adquirir hatos propios, aunque el monopolio de los patrones sobre los canales de comercialización aseguraba su posición dominante (Santos-Granero y Barclay 1995). Por otro lado, las tierras que ocupaban fueron transformándose crecientemente en pastizales para ganadería.

Las marcas de estos cambios pueden hallarse en la C.N. Siete de junio. La creación política de su sector principal, Villa América, era reconocida por los comuneros en el año de 1960; y según lo conversado con los comuneros más longevos, el espacio de la comunidad no se hallaba poblada inicialmente por muchas familias. Sin embargo, Smith (1982) señala que la comunidad ya se hallaba conformada por 94 familias en el año de 1968, y concentraba la mayor cantidad de ganado vacuno entre las comunidades del Medio Palcazú. En adición, este espacio del valle se vio presionado por la extracción selectiva de madera, impulsada por comerciantes madereros de la región durante ciertos momentos del siglo pasado (Benavides y Pariona 2002). De esta manera, el proceso de inserción a la economía de mercado de los yánesha del Medio Palcazú, marcado por la presión sobre sus recursos naturales, ha constituido el marco general para la transformación ambiental de esta parte de la cuenca.

A inicios de la década de 1980, el gobierno de Fernando Belaúnde emprendió la búsqueda por concretar sus políticas de desarrollo agropecuario en la Amazonía mediante los proyectos especiales en selva. En el caso del futuro Proyecto Especial Pichis Palcazú (PEPP), se tuvo una oposición activa de varios actores a esta formulación original, fundamentándose en las consecuencias negativas que podrían atravesar del valle (ocupación desmedida y deterioro ambiental) ante la colonización promovida por una carretera (Smith 1982; Santos-Granero y Barclay 1995). Ante esta campaña, la Agencia de los Estados Unidos para el Desarrollo Internacional (USAID), la entidad financiadora de la parte del proyecto dirigida al Palcazú, decidió fijar las condiciones para establecer un proyecto orientado al manejo de recursos naturales. El diseño de sus componentes, a su vez, enfatizó el beneficio y la participación de la población yánesha de las comunidades del valle (Hartshorn y Stocks 1993; Staver et al. 1994).

Bajo esta nueva dirección, el PEPP continuó la titulación de comunidades nativas en el Palcazú, un proceso iniciado a mediados de la década de 1970. Otras intervenciones a gran escala que involucraron al PEPP fueron el establecimiento del Parque Nacional Yanachaga Chemillén (1986), el Bosque de Protección San Matías - San Carlos (1987) y la Reserva Comunal Yanesha (1988). Además, se organizó la Cooperativa Forestal Yanesha como iniciativa para el aprovechamiento forestal comunitario desde 1986, aunque no pudo sostenerse en los años posteriores a su creación (Hartshorn y Stocks 1993; Staver et al. 1994; Benavides y Pariona 2002). Al aplicarse este conjunto de medidas, se pudo evitar la ocupación desmedida y la degradación ambiental que el valle del Palcazú podría haber experimentado debido a las presiones de colonización que ya se estaban experimentando en la selva central desde la década pasada (Santos-Granero y Barclay 1995). Aunque las medidas ambientales del PEPP han asegurado la propiedad colectiva de los yánesha sobre su territorio y ha mitigado la deforestación de los bosques de la cuenca, la construcción de la carretera Villa Rica - Iscozacín facilitó el ingreso de nuevos actores al valle y ha promovido cambios en la vida social y económica de los yánesha ${ }^{3}$ (Salick, 1989; Hamlin y Salick 2003; Larsen 2016).

\footnotetext{
${ }^{3}$ La historia de estas tensiones alrededor de los componentes de áreas protegidas y titulación comunal, por un lado, y la carretera, por el otro, ha sido escrita en detalle por autores como Smith (1982), Santos-Granero y Barclay (1995), y Larsen (2016).
} 

en la Comunidad Nativa Siete de junio, Perú

Entre las décadas de 1980 y 1990 el valle de Palcazú y sus habitantes experimentaron la violencia política por parte de grupos subversivos, además de la intensificación de la extracción forestal selectiva y depredadora, y la entrada de grupos narcotraficantes (Santos-Granero y Barclay 1995). Así, los proyectos para actividades productivas sostenibles se detuvieron durante algunos años. No obstante, a partir del 2000 volvieron a proliferar programas y proyectos bajo los esquemas del desarrollo sostenible. Esto, a su vez, conllevó al incremento de las asociaciones, conformadas por los comuneros yánesha, y orientadas a la realización de las actividades sostenibles insertadas (Valadeau 2016). Entre estas últimas, el cultivo comercial del cacao y la piscicultura han ido ganando mayor terreno. Como se verá después, las nuevas formas asociativas constituyen el nuevo campo de encuentro de las prácticas y discursos de las intervenciones ambientales con la organización social yánesha existente.

\section{Cambios recientes en el uso de la tierra}

En las últimas dos décadas, el uso de la tierra en el distrito de Palcazú ha ido cambiando paulatinamente con el ingreso de las nuevas actividades sostenibles. Aunque también se considera la superficie de pastizales ocupadas por medianos y grandes propietarios particulares no indígenas, la comparación de los datos sobre el uso de la tierra entre los años 2000 y 2016 (Geobosques 2019) ayuda a pensar acerca de ciertos cambios y continuidades para las CC.NN. como Siete de junio. En el Cuadro 1 se pueden observar los cambios en la superficie destinada a diferentes usos de la tierra a nivel del distrito. En el año 2016, la superficie del bosque disminuyó en un $9.5 \%$ respecto a la superficie del año 2000, pasando a comprender el $73.9 \%$ de las tierras del distrito. Es decir, la mayoría de las tierras en el Palcazú están cubiertas por bosques, mientras que un $23.4 \%$ comprende a las tierras de uso agropecuario (tierras agrícolas, bosques secundarios o purmas, y pastizales para ganadería). Para el año 2016, estas tierras incrementaron su superficie aproximadamente un $49 \%$ respecto a la exten- sión que tenían en el año 2000. Sin embargo, las proporciones específicas de cada uno de sus componentes han variado sutilmente. Si bien el porcentaje de purmas resulta casi idéntico (alrededor del 64\%), las tierras agrícolas han incrementado de un $10.4 \%$ a un $16.4 \%$, mientras que los pastizales han disminuido de un $24.7 \%$ a un $20.2 \%$. La interpretación para estos cambios requiere una aproximación más minuciosa.

A partir de la información sobre cambios en el uso de la tierra disponibles en la misma plataforma (Cuadro 2), se observa que entre los años 2000 y 2016, la conversión del conjunto de otras categorías de otros usos de la tierra a la de pastizales fue de 5,970 hectáreas, mientras que la conversión de superficie en la dirección opuesta fue de 3,618 hectáreas, resultando en un incremento neto de 2,352 hectáreas de pastizal. Sin embargo, al desagregarlo por periodos, puede constatarse que el incremento en la conversión a pastizales disminuye abruptamente a partir del 2011, manteniéndose de esta manera hasta el 2016. Contrastando con esto, se observa un crecimiento súbito en el mismo periodo en las tierras con usos agrícolas, resultando en un incremento neto de 6,154 hectáreas. No obstante, los datos de Geobosques (2019) señalan que el $88.3 \%$ de este incremento provino de las tierras cuya superficie estaba constituida por bosques primarios y secundarios.

¿Cómo interpretar la disminución de los pastizales y la duplicación de la superficie con usos agrícolas? A nivel de todo el distrito, el incremento en la presión poblacional sobre los recursos no parecer ser una respuesta adecuada a esta pregunta. Comparando la población de Palcazú entre los últimos dos censos de población, se constata un descenso del 19\% entre los años 2007 y 2017. No obstante, la población de las comunidades nativas ha ido incrementando aproximadamente en un $41 \%$ durante el mismo periodo (INEI 2008, 2018). Se sugiere que el crecimiento demográfico en las comunidades yánesha, circunscrita a una cantidad determinada de tierras, podría haber impulsado la apertura de nuevas chacras en bosques primarios y secundarios. Por otro 


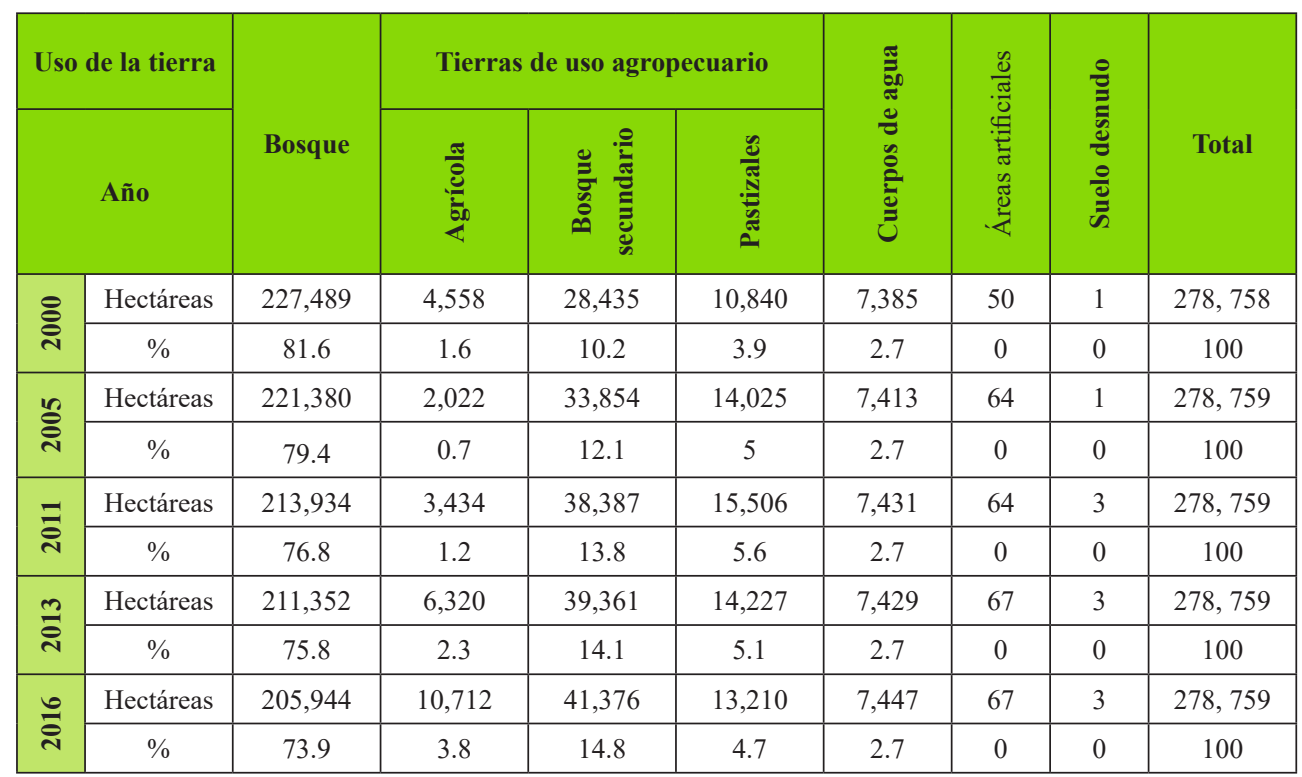

Cuadro 1. Usos de la tierra - Palcazú (2000-2016). Elaboración del autor con base en Geobosques (2019).

\begin{tabular}{|c|c|c|c|c|c|c|}
\hline \multicolumn{2}{|c|}{ Usos de la tierra } & $2000-2005$ & 2005-2011 & 2011-2013 & 2013-2016 & Totales \\
\hline \multirow{3}{*}{ 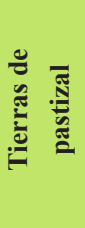 } & Incremento $(+)$ & 3,185 & 2,275 & 226 & 293 & 5,970 \\
\hline & Disminución (-) & 0 & 793 & 1,505 & 1,311 & 3,618 \\
\hline & $\begin{array}{c}\text { Incremento o } \\
\text { disminución neta }\end{array}$ & $3,185(+)$ & $1,482(+)$ & $1,279(-)$ & $1,018(-)$ & $2,352(+)$ \\
\hline \multirow{3}{*}{ 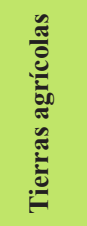 } & Incremento $(+)$ & 353 & 1,429 & 3,436 & 5,377 & 10,595 \\
\hline & Disminución (-) & 2,889 & 18 & 550 & 984 & 4,441 \\
\hline & $\begin{array}{c}\text { Incremento o } \\
\text { disminución neta }\end{array}$ & $2,536(-)$ & $1,411(+)$ & $2,886(+)$ & $4,393(+)$ & $6,154(+)$ \\
\hline
\end{tabular}

Cuadro 2. Cambios en el uso de las tierras agrícolas y pastizales - Palcazú (2000-2016). Elaboración del autor con base en Geobosques (2019).

lado, su vida económica ha sido intervenida de forma particular por los proyectos de desarrollo sostenible. Desde inicios del 2000, las actividades productivas propuestas por los proyectos de desarrollo, como el cultivo comercial del cacao, se han basado en la premisa de sustituir a otras actividades comerciales que contribuían en mayor medida a la degradación ambiental del valle (Hartshorn y Stocks 1993; Staver et al. 1994). De forma especial, estas se han diseñado e implementado con base en el modelo del desarrollo alternativo, cuyo objetivo principal es evitar la expansión de la producción de hoja de coca para el narcotráfico. 

en la Comunidad Nativa Siete de junio, Perú

En la C.N. Siete de junio se puede visualizar el cambio en el uso de la tierra y la presión sobre los bosques de una población que ha incrementado constantemente en el transcurso de cinco décadas. Como se vio líneas atrás, la creación política de Siete de junio antecede a su cristalización bajo la forma socioespacial de la Comunidad Nativa, siendo titulada poco después de la promulgación del D.L. 20653 en el año de 1975 (IBC 2006). Según el III Censo de Comunidades Indígenas, su población se encontraba conformada por 1,194 habitantes (INEI 2018). Durante la realización del trabajo de campo, la misma se encontraba distribuida alrededor de 12 sectores, los cuales constituyen las unidades sociopolíticas básicas de la comunidad. Cada sector, a su vez, se encontraba formado por el conjunto de los comuneros, los hombres y mujeres adultos con atribuciones para la toma de decisiones en la comunidad y con responsabilidades asumidas respecto al sector.

Con una superficie de 8,782 hectáreas, la C.N. Siete de junio cuenta con la mayor extensión entre las comunidades del valle (IBC 2006). Ubicado en el suelo del valle, se encuentra atravesado por los ríos Omaiz y Comparachimás (antes de formar el río Chuchurras aguas abajo). Las tierras, por su parte, pueden dividirse en las categorías amplias de terrenos aluviales (o pampa) y terrenos de altura, siguiendo el sistema de clasificación yánesha de la tierra registrado en la década de 1980 por Jan Salick (1989). De igual manera, estas pueden clasificarse de acuerdo con su capacidad de uso mayor (Smith 1982). En el Cuadro 3, estos últimos datos han sido adaptados con información censal proveniente de periodos posteriores al estudio original (IBC 2006; INEI 2018), permitiendo visualizar la cantidad de superficie agropecuaria promedio que los comuneros dispondrían actualmente.

La superficie agrícola de la comunidad, compuesta por la suma de las tierras con capacidad de uso mayor para cultivos en limpio (A), cultivos permanentes (C) y pastizales (P), conforman el $39.5 \%$ de la superficie total de la comunidad. En 1968, esto implicaba que una familia yánesha de cinco miembros tenía en promedio 36.5 hectáreas de superficie agrícola. Sin embargo, el crecimiento demográfico al interior de la comunidad ha ido disminuyendo el promedio de tierras disponible para cada familia en las décadas siguientes. La presión sobre las tierras disponibles alcanzó un punto máximo en el año 2000, con un promedio de 13.8 hectáreas por familia. Aunque esta proporción ha incrementado ligeramente en el

\begin{tabular}{|c|c|c|c|c|c|c|c|c|}
\hline \multirow{2}{*}{$\begin{array}{c}\text { Clases de } \\
\text { uso de la } \\
\text { tierra }\end{array}$} & Total de & \multirow{2}{*}{ \%ectáreas } & \% Total & \multicolumn{7}{|c|}{ Hectáreas/Familia } \\
\cline { 5 - 9 } & $\mathbf{1 9 6 8}$ & $\mathbf{1 9 7 6}$ & $\mathbf{1 9 8 0}$ & $\mathbf{1 9 9 0}$ & $\mathbf{2 0 0 0}$ & $\mathbf{2 0 1 7}$ \\
$\mathbf{A}$ & 395 & 4.5 & 4.2 & 3.8 & 3.4 & 2.2 & 1.6 & 1.7 \\
\hline $\mathbf{P}$ & 1,580 & 18 & 16.6 & 15.2 & 13.6 & 8.8 & 6.3 & 6.6 \\
\hline $\mathbf{C}$ & 1,493 & 17 & 15.7 & 14.4 & 12.9 & 8.3 & 5.9 & 6.2 \\
\hline $\mathbf{F 1}$ & 3,073 & 35 & 32.3 & 29.5 & 26.5 & 17.1 & 12.2 & 12.9 \\
\hline $\mathbf{F 2}$ & 1,317 & 15 & 13.9 & 12.7 & 11.4 & 7.3 & 5.2 & 5.5 \\
\hline $\mathbf{X}$ & 924 & 10.5 & 9.7 & 8.9 & 8.0 & 5.1 & 3.7 & 3.9 \\
\hline Total & $\mathbf{8 , 7 8 2}$ & $\mathbf{1 0 0}$ & $\mathbf{9 2 . 4}$ & $\mathbf{8 4 . 4}$ & $\mathbf{7 5 . 7}$ & $\mathbf{4 8 . 8}$ & $\mathbf{3 5 . 0}$ & $\mathbf{3 6 . 7}$ \\
\hline
\end{tabular}

Cuadro 3. Superficie promedio por familia de comuneros según las capacidades de uso mayor de la tierra en la C.N. Siete de junio (1968 - 2017). Elaboración del autor con base en IBC (2006), INEI (2018) y Smith

(1982). 


\begin{tabular}{|c|c|c|}
\hline Comunidad Nativa & $\begin{array}{l}\text { Hectáreas de bosque a } \\
\text { conservar con TDC* }\end{array}$ & $\begin{array}{c}\text { Monto asignado por la } \\
\text { TDC (en soles) }\end{array}$ \\
\hline Alto Iscozacín & 1,400 & 14,000 \\
\hline Alto lagarto & 1,100 & 11,000 \\
\hline Buenos Aires & 2,100 & 21,000 \\
\hline Nueva Esperanza & 3,649 & 36,490 \\
\hline Santa Rosa de Chuchurras & 900 & 9,000 \\
\hline Santa Rosa de Pichanaz & 1,000 & 10,000 \\
\hline Shiringamazú & 1,100 & 11,000 \\
\hline Total & 11,249 & 112,490 \\
\hline
\end{tabular}

Cuadro 4. Comunidades Nativas de Palcazú con convenios vigentes con el Programa Bosques (octubre 2019). Elaboración del autor con base en PNCBMCC (2019).

año 2017, con 14.5 hectáreas por familia, sigue siendo menos de la mitad de aquella que era disponible en 1980.

Considerando el caso ideal en el que todas las familias acceden a este promedio equitativamente, la superficie agrícola se encuentra por debajo de la cantidad mínima estimada para la continuidad óptima de la horticultura de subsistencia, con el periodo de barbecho existente en la década de 1980 (Smith 1982). Esta actividad también se vería afectada por la dedicación de los comuneros a otras actividades proveedoras de ingresos monetarios dentro o fuera de las tierras de la familia (Hamlin y Salick 2003). Por otro lado, este promedio distorsiona la distribución real de la tierra dentro de la comunidad por dos motivos. El primero consiste en que las tierras de las diferentes familias y sectores se hallan ubicadas en diferentes tipos de terrenos. En el caso del sector de Pampacocha, donde se enfocó el trabajo de campo, se constató que su ubicación en terrenos aluviales, en las riberas del río Omaiz, permite una mayor dedicación a cultivos de subsistencia, cultivos permanentes (cacao) y, en menor medida, crianza de ganado. En segundo lugar, debe tenerse en cuenta la existencia de desigualdades en la tenencia de la tierra entre las familias, usualmente determinadas por la historia de ocupación de la comunidad (Hartshorn y Stocks 1993). Estas diferencias pudieron constatarse en el trabajo de campo, a través de las conversaciones y observaciones sobre las actividades de cultivo entre las familias de Pampacocha ${ }^{4}$.

A partir de lo expuesto, puede afirmarse que los yánesha del Palcazú han incrementado la superficie bajo uso agrícola debido al incremento de la población, prefiriendo adoptar las actividades de cultivo comercial de cacao, insertadas por los proyectos de desarrollo sostenible en el transcurso del siglo XXI, como fuente de ingresos monetarios. Un primer motivo de esta preferencia radica en la promoción activa de su instalación por parte del Programa de Desarrollo Alternativo en las Áreas de Pozuzo Palcazú (PRODAPP) y, posteriormente, la Comisión para el Desarrollo y Vida sin Drogas (DEVIDA). Otro motivo radica en la preferencia declarada por los comuneros de Siete de junio para el cultivo del cacao con respecto a la ganadería. Esta última resulta especialmente tediosa por la demanda de insumos y tiempo para la crianza de vacunos, entrando en tensión con la multiplicidad de actividades

${ }^{4} \mathrm{~A}$ pesar de no contar con mediciones, el uso de estas técnicas permitió observar la mayor disponibilidad de tierras que disponía una de las familias consignadas en el estudio de Staver (1989). 

en la Comunidad Nativa Siete de junio, Perú

productivas cotidianas de los yánesha, además de constreñir el rango de actividades sociales recurrentes en la comunidad. No obstante, se constata la continuidad y la yuxtaposición de tierras para el cultivo del cacao y para la crianza de hatos de ganado vacuno en las tierras aluviales, adyacentes al río Omaiz y sus quebradas, pertenecientes al sector de Pampacocha. Aun así, estos pastizales no cubren grandes extensiones continuas (como es el caso de las zonas circundantes al río Chuchurras).

\section{Intervenciones ambientales para la conser- vación y desarrollo sostenible}

Como puede sostenerse a partir de las líneas anteriores, la historia de la relación entre los bosques del Palcazú y sus habitantes yánesha ha estado marcada por la inserción al mercado de estos últimos. En concordancia con investigaciones pasadas (Staver 1989; Hamlin y Salick 2003), se observó que, en el caso del sector Pampacocha, las actividades productivas para el sustento local y comercial se han concentrado en los terrenos aluviales. Mientras tanto, algunas secciones de las tierras de altura son abiertas por los comuneros con la finalidad de abrir chacras o extraer madera para la construcción de viviendas. Los comuneros y el personal de proyectos, empleando sus respectivos entendimientos del entorno de la comunidad, han descrito los bosques o el monte circundantes como espacios distribuidos de manera irregular, así como relativamente menores en comparación a los de otras comunidades. En este contexto, el ingreso de las intervenciones ambientales, como el establecimiento de las áreas protegidas y proyectos para el manejo de los recursos forestales, han marcado nuevas direcciones para los yánesha y sus instituciones involucradas en el uso de los bosques comunitarios.

Hacia el año 2019, el uso de la tierra de los habitantes yánesha del Medio Palcazú se encontraba intervenido, en cierta medida, por una serie de instituciones estatales y privadas. En Iscozacín, el poblado capital, las instituciones estatales ambientales concentran a los funcionarios, artefactos e infraestructuras que las componen. Estos son el Servicio $\mathrm{Na}$ cional Forestal y de Fauna Silvestre (SERFOR), que cumple con sus atribuciones reguladoras a través de la oficina desconcentrada de la Administración Técnica Forestal y de Fauna Silvestre (ATFFS); y el Servicio Nacional de Áreas Naturales Protegidas (SERNANP), que cogestiona la RCY junto a AMARCY. Esta última también cuenta con una oficina, albergada dentro del edificio de SERNANP. De la misma manera, la ONG Desarrollo Rural Sustentable (DRIS) tiene una oficina propia para facilitar sus actividades.

Entre los meses de abril y mayo de aquel año, DRIS se encontraba articulando proyectos con el objetivo expreso de fortalecer la cogestión de la Reserva Comunal Yánesha, secundando a AMARCY y las organizaciones yánesha pertenecientes a sus comunidades socias. Bajo el esquema que rige a estos proyectos, diferentes instituciones yánesha eran incorporadas activamente en la formulación y ejecución de tales intervenciones. Así, DRIS ha apoyado la consolidación progresiva de AMARCY en la toma de decisiones para la cogestión de la RCY. De igual manera, esta ONG ha apoyado la suscripción de los miembros de las comunidades socias de AMARCY al Programa Nacional de Conservación de Bosques en los últimos años, enfocándose en la institución de la junta directiva comunal. Por otro lado, DRIS también ha apoyado a la Asociación de Productores Agropecuarios y Forestales Sancore (o Asociación Sancore), formada por miembros de la C.N. Siete de junio $^{5}$, mediante la facilitación de su postulación a un fondo concursable. Abordar estas iniciativas permite entender los resultados de las intervenciones ambientales para la conservación entre los yánesha en los últimos cinco años.

\footnotetext{
${ }^{5}$ Esta asociación, formada a inicios de la década del 2000 (Valadeau 2016), se encontraba formada por comuneros de Pampacocha y otros sectores de la comunidad. Durante la realización del trabajo de campo, la participación de los comuneros socios había disminuido a raíz del descontento que causaron las irregularidades de un fondo concursable anterior.
} 
El apoyo de DRIS a AMARCY como cogestora de la RCY se ha basado en el fortalecimiento de la autonomía de esta institución. A partir de la facilitación de alianzas institucionales y la captación de fondos monetarios de fuentes bilaterales o internacionales (como el PNUD), la ONG persiguió el objetivo de que AMARCY gestionase sus recursos para llevar a cabo su agenda. En consecuencia, la asociación ha podido sostener asambleas con los líderes de las comunidades y anexos colonos asociados. Durante una de estas asambleas, realizada en el sector Conaz a fines de mayo del 2019, se tocaron temas relativos a la labor de SERNANP, los proyectos de cacao apoyados por DRIS y el problema de la ocupación y deforestación de la reserva por parte de colonos invasores. Este último tema acaparó la atención de los líderes participantes, y suscitando la enérgica propuesta de desalojo de los invasores por parte de un jefe de comunidad. Si bien estas declaraciones en la asamblea deben ser entendidas a la luz de las dinámicas sociopolíticas existentes entre los yánesha (las cuales escapan al tema de este texto), también proveen evidencia de que los actores de las instituciones indígenas locales pueden alinearse junto a los objetivos de las intervenciones ambientales (aunque parcialmente), volviéndose partícipes de estas.

La suscripción del convenio del Programa Bosques para las comunidades socias de AMARCY, por su parte, se basó en la articulación de la junta directiva con el programa de conservación. Para lograr esto, DRIS facilitó el sostenimiento de reuniones entre los funcionarios del programa con los líderes y representantes de las comunidades participantes. En una entrevista con el ingeniero a cargo de la oficina de DRIS en Palcazú, este señaló que las comunidades socias de AMARCY originalmente no eran contempladas como beneficiarias debido a los requisitos de superficie (en hectáreas) de bosque continuo que estipulaban los funcionarios del programa. Como se constató en la sección anterior, los bosques comunitarios del Palcazú se encuentran irregularmente distribuidos debido a la conversión de las tierras para usos hortícolas, agrícolas o ga- naderos; y han experimentado la deforestación de las tierras (principalmente aluviales) para estas actividades. A pesar de esta desventaja, la ONG persuadió a los funcionarios señalando la cualidad que las comunidades socias adquirían como la zona de amortiguamiento de la RCY. Respaldadas por las 34,774 hectáreas de la reserva, el resultado ha sido la suscripción del Programa Bosques para nueve de las diez comunidades socias (Cuadro 4).

Además de los compromisos asumidos por las juntas directivas comunales para la conservación de los bosques comunitarios, estas también han adquirido la responsabilidad de invertir el monto obtenido por el mecanismo de la Transferencia Directa Condicionada (TDC) en actividades productivas regidas bajo esquemas de desarrollo sostenible. Según lo conversado con el jefe de la oficina local de DRIS, esta inversión requiere una planificación previa a su ejecución. Este es el caso de la C.N. Nueva Esperanza, cuya superficie de bosque conservada es la mayor entre las CC.NN. socias. Ubicada al oeste de Siete de junio, sobre tierras más próximas a la RCY, los bosques comunitarios y recursos forestales de Nueva Esperanza han sido menos intervenidas por actividades productivas, pudiendo obtener 36,490 soles anuales a través de la TDC. Una mañana de abril, durante el trabajo de campo, un comunero de Pampacocha se preparaba para dirigirse junto a un compañero de otro sector a la comunidad aledaña de Nueva Esperanza con el fin de llevar a cabo labores de instalación de plantones de cacao. Mientras lo esperaba, me contó que el servicio de instalación había sido solicitado por la 'gente del proyecto bosques'.

Si bien no pudo concretarse el trabajo de instalación en aquella ocasión, pudo obtenerse un mayor entendimiento sobre las implicaciones del encauzamiento de las decisiones económicas efectuadas por la comunidad, a raíz de la intervención ambiental del Programa Bosques. Este, además de perseguir objetivos explícitos de conservación, también permitía su retroalimentación con los objetivos de desarrollo sostenible al canalizar los montos del TDC hacia actividades como el cultivo comer- 

en la Comunidad Nativa Siete de junio, Perú

cial del cacao. En el camino, la junta directiva gana atribuciones y responsabilidades en el manejo de estos fondos monetarios; y los habitantes yánesha que aprendieron tempranamente las técnicas para el cultivo del cacao llevan a cabo las actividades de instalación de cultivos. Mientras tanto, DRIS y el Programa Bosques asumen roles de asistencia técnica y capacitación.

El apoyo de DRIS a la Asociación Sancore mediante las intervenciones para desarrollo sostenible se enlaza en formas sutiles con las intervenciones para conservación. Específicamente, la ONG ha otorgado asistencia técnica y capacitación a los socios de Sancore durante la formulación, postulación y ejecución de un proyecto para mantenimiento y comercialización de cacao a través de un fondo concursable, el Procompite regional. Además de los objetivos de desarrollo sostenible basados en la continuidad del cultivo del cacao (entendido como un mitigador de la deforestación por actividades agrícolas comerciales), DRIS busca (como con las otras intervenciones) la mayor autonomía de la Asociación Sancore. Por otro lado, según lo conversado con un expresidente de Sancore, la asociación habría sostenido un acuerdo con SERNANP que involucraba la participación de sus miembros (como él) en labores de vigilancia comunal de bosques. De esta manera, se evidencia que la Asociación Sancore busca ser encauzada como una institución cuyos miembros, los comuneros yánesha, usen para relacionarse con el mercado mientras se persiguen objetivos ambientales.

En esta gama de intervenciones ambientales, la C.N. Siete de junio provee un caso interesante, donde los objetivos buscados por las intervenciones de DRIS y las instituciones estatales ambientales no se consiguen de forma absoluta. En el caso de las intervenciones ambientales para conservación, se halló que Siete de junio fue la única C.N. socia que no suscribió el convenio con el Programa Bosques. Esto se debió principalmente a los desacuerdos surgidos entre los comuneros a cargo de la toma de decisiones, a raíz de diferentes interpretaciones de las declaraciones de los funcionarios del
Programa Bosques en la asamblea comunal. Según lo conversado con dos comuneros, algunos líderes se opusieron al otorgamiento de S/ 10 por hectárea de bosque conservado, argumentando que el monto debía ser más elevado. Según estos recuentos, estas personas asumieron que el monto sería distribuido según la cantidad de superficie de bosque con el que contaba cada familia, en lugar de la superficie de bosque de toda la comunidad. Asimismo, el jefe de la oficina zonal de DRIS mencionó que algunos comuneros se opusieron a la suscripción del convenio al asociar el término 'plan de vida' (un requerimiento de las comunidades) con DEVIDA, la institución que actualmente lidera la promoción del cacao como cultivo alternativo a la hoja de coca. El comentario del comunero en cuestión aludía a la intromisión de esta institución en la autonomía de las decisiones productivas de los comuneros.

\section{Discusión}

A partir de lo expuesto en la sección anterior, se discutirán brevemente dos temas. El primero concierne a la influencia del contexto de la economía política sobre el curso de las intervenciones ambientales. $\mathrm{Al}$ igual que el proyecto de manejo de recursos del PEPP y USAID, los proyectos de cacao de inicios del 2000 se basaban en la promoción de una actividad ambientalmente sostenible. No obstante, el trasfondo de estos últimos ha consistido en la oposición a la expansión de los cultivos de hoja de coca vinculados a las actividades de narcotráfico en la región. En este sentido, puede afirmarse que incluso los proyectos formulados fuera del esquema del 'desarrollo alternativo' pueden tener efectos más allá del campo ambiental. Los programas y proyectos basados en la conservación o aprovechamiento forestal, en contraste, han sido escasos, cobrando una mayor relevancia con el advenimiento del Programa Bosques en la última década.

El segundo tema trata acerca de los conceptos de environmentality y ecogubernamentalidad, originados a partir de la elaboración foucaultiana de governmentality, y desplegados en la dimensión de prácticas y discursos 
ambientales. Un argumento esgrimido por Cepek (2011) ha llamado la atención sobre los peligros de malinterpretar los programas y proyectos ambientales como fuerzas que moldean unidireccionalmente al 'sujeto ambiental' buscado. Con base en el desarrollo de las intervenciones ambientales de DRIS sobre las instituciones indígenas asociadas a la RCY durante los últimos cinco años, y el caso particular de la C.N. Siete de junio, sostengo que las intervenciones ambientales no deben entenderse como transformadoras definitivas de la subjetividad de los comuneros yánesha hacia un 'sujeto ambiental. Más bien, las nuevas prácticas y discursos ambientales son incorporados parcialmente y reconfigurados (o incluso contestados abiertamente) al ingresar entre los miembros de las diferentes instituciones (asociación para el manejo de la reserva, junta directiva, asociación de productores) enlazadas con los programas y proyectos para conservación y desarrollo sostenible.

\section{Conclusiones}

A lo largo de este texto, se ha buscado responder la pregunta sobre las intervenciones para la conservación de los bosques de las comunidades nativas del Medio Palcazú durante los últimos cinco años. A través de los enfoques escogidos, se ha procurado proveer una descripción e interpretación sobre los cambios en los usos de la tierra del distrito y las intervenciones para conservación de la ONG DRIS y el Programa Bosques. La información cualitativa recogida y expuesta permite afirmar que la comunidad se halla permeada por la combinación de actividades productivas para el sustento local y para el comercio. Entre estas últimas, puede concluirse que el contexto socioambiental de Siete de junio está caracterizado por la preferencia creciente de los comuneros por proveer sus ingresos monetarios a través del cultivo comercial del cacao. En otras comunidades, por su parte, los ingresos provenientes de las transferencias directas condicionadas del Programa Bosques son canalizadas por la junta directiva. No obstante, esta puede vincularse indirectamente a la economía fami- liar a través de la promoción de actividades productivas sostenibles.

En contraste con las otras comunidades socias de AMARCY, presentadas como casos exitosos de los programas y proyectos de conservación ambiental y desarrollo sostenible, el caso particular de la C.N. Siete de junio permitió obtener un entendimiento más cabal sobre los alcances y las limitaciones de estas intervenciones. En Siete de junio se tenía un contexto caracterizado, en el momento, por una menor disposición de los comuneros para organizar y llevar a cabo actividades orientadas a la conservación de bosques comunitarios autónomamente mediante las formas institucionales existentes (sean antiguas o nuevas). Esto dificulta la persecución de los objetivos ambientales que pautan los programas y proyectos que proliferan en el Palcazú. No obstante, los esfuerzos articulados entre la población yánesha y las instituciones estatales y privadas demuestran que es posible fomentar la formación de instituciones que puedan manejar adecuadamente los bosques comunitarios. No obstante, siempre debe advertirse sobre el peligro de alienar a los comuneros participantes respecto a su historia de relaciones con el entorno y de su vida social.

A partir de este estudio, se esbozan tres recomendaciones para las futuras intervenciones orientadas a la conservación de los entornos biofísicos habitados por los yánesha del Palcazú. Las mismas se encuentran dirigidas hacia los actores institucionales (estatales o privados no indígenas) que actualmente canalizan los fondos y tienen el mayor margen de decisión en los programas y proyectos. En primer lugar, se recomienda que los diseños de estos últimos incorporen las motivaciones detrás del comportamiento económico y ecológico de la población yánesha. Estos últimos no deberían excluir el papel de factores económico-políticos, sociales y culturales a escalas locales, regionales o más amplias. Así, la retroalimentación posible entre las TDC del Programa Bosques y las actividades productivas promovidas por los proyectos de desarrollo sostenible, descrita en líneas anteriores, es un ejemplo de una sinergia potencialmente eficaz. 

en la Comunidad Nativa Siete de junio, Perú

En segundo lugar, y en la línea de lo anterior, también se recomienda la consideración de la variabilidad del entorno biofísico accesible para cada familia de comuneros yánesha. En este sentido, puede observarse que las intervenciones para el desarrollo sostenible (especialmente basadas en el cultivo del cacao) han avanzado de una forma más sensible al contexto, considerando la composición del conjunto de actividades productivas de los yánesha del Palcazú. Sin embargo, los yánesha del sector de Pampacocha cuentan con mayor disponibilidad de tierras aluviales que otros sectores; $y$, asimismo, han sido beneficiados en mayor medida por intervenciones pasadas. La noción de intervención ambiental, explorada en este texto, apunta a abrir una reflexión y una crítica sobre la persecución de un horizonte de objetivos ambientales, los cuales se desconectan del contexto histórico, sociopolítico y ecológico que permea la relación entre los habitantes del bosque y este mismo.

Por último, se recomienda una mayor apertura y flexibilidad por parte de las instituciones estatales ambientales para articular sus iniciativas de conservación ambiental con la población yánesha (e indígena en general). Aquellas deben tomar en cuenta un contexto muy variable para el estado de las instituciones indígenas consideradas en los diseños de sus programas y proyectos. A pesar de que puede trabajarse con las CC.NN. socias de AMARCY mediante sus instituciones comunales y asociaciones de productores, estas últimas se diferencian con base en su forma de organización sociopolítica, su tamaño, la disposición de sus miembros a actualizar las actividades de la institución, entre otras características. De esta forma, se concluye que el futuro en la conservación y uso adecuado de los bosques en el valle del Palcazú dependerá de los esfuerzos colectivos de sus habitantes para concretar estas metas; $y$, entre estos, los yánesha demuestran contar con un gran potencial para organizarse para el uso y cuidado de los bosques de su territorio.

\section{Agradecimientos}

Este estudio se enmarcó en el Proyecto de investigación aplicada: "Cambios y permanen- cias en el manejo de bosques y usos de la tierra por pueblos indígenas amazónicos: Estudios de caso en comunidades nativas asháninka y yánesha - FIFA". Por tal motivo, el autor agradece el apoyo proporcionado por SERFOR CAF a través del financiamiento del trabajo de campo.

De igual manera, el autor agradece de todo corazón a los comuneros de Pampacocha por la hospitalidad y apertura a la realización del trabajo de campo entre ellos.

\section{Bibliografía}

Agrawal, A. 2005. Community, Intimate Government, and the Making of Environmental Subjects in Kumaon, India. Current Anthropology 46(2): 161-190.

Benavides, M; Pariona, M. 2002. La cooperativa forestal Yanesha y el sistema de manejo forestal comunitario en la selva central peruana. In Smith, RC; Pinedo, D. (eds.). El cuidado de los bienes comunes: gobierno y manejo de los lagos y bosques en la Amazonía. Lima, Perú, IEP. p. 305-333.

Cepek, ML. 2011. Foucault in the forest: Questioning environmentality in Amazonia. American Ethnologist 38(3): 501-515.

Geobosques. 2019. Cambio de uso de tierra (en línea). Lima, Perú, PNCBCC. Disponible en http://geobosques.minam.gob.pe/geobosque/ view/cambio-uso.php.

Hamlin, C; Salick, J. 2003. Yanesha agriculture in the upper peruvian Amazon: Persistence and change fifteen years down the 'road'. Economic Botany 57(2): 163-180.

Hartshorn, G; Stocks, A. 1993. The Palcazu project: Forest management and native Yanesha communities. Journal of Sustainable Forestry 1(1): 111-135.

IBC (Instituto del Bien Común) (ed.). 2006. Atlas de comunidades nativas de la selva central. Lima, Perú. 115 p.

IBC (Instituto del Bien Común). 2020. Visualizador de mapas (en línea). Lima, Perú. 
Consultado 26 set. 2020. Disponible en http://191.98.188.187/ibcmap.

INEI (Instituto Nacional de Estadística e Informática). 2008. Censos Nacionales 2007: XI de Población y VI de Vivienda: resultados definitivos de comunidades indígenas. Lima, Perú. v. 1, 621 p.

INEI (Instituto Nacional de Estadística e Informática). 2018. Directorio de Comunidades Nativas y Campesinas. Censos Nacionales 2017: XII de Población, VII de Vivienda y III de Comunidades Indígenas. III Censo de Comunidades Nativas y I Censo de Comunidades Campesinas. Lima, Perú. v. 1, 478 p.

Larsen, PB. 2016. Derechos indígenas, gobernanza ambiental y recursos en la Amazonía peruana: hacia una antropología de la posfrontera. Lima, Perú, IEP. 275 p.

Luján Berger, S. 2017. Una aproximación al cambio en el uso de los recursos y las actividades económicas: La pesquería y la ganadería en la Comunidad Nativa «Loma Linda» del distrito de Palcazu-Pasco. Tesis Lic. Lima, Perú, Pontificia Universidad Católica del Perú. $153 \mathrm{p}$.

PNCBMCC (Programa Nacional de Conservación de Bosques). 2019. Comunidades nativas socias del Programa Bosques con convenios vigentes - octubre 2019 (en línea). Lima, Perú. Consultado 15 ene. 2020. Disponible en http://www.bosques.gob.pe/archivo/CCNNal-310CT19.pdf.

Salick, J. 1989. Bases ecológicas de los sistemas agrícolas Amuesha. Amazonía Indígena 9(15): 3-16.

Santos-Granero, F. 2004. Los Yánesha. In Santos, F; Barclay, F (eds.). Guía Etnográfica de la Alta Amazonía. Balboa, Panamá, Smithsonian Tropical Research Institute. v. 4, p. 159-359.

Santos-Granero, F; Barclay Rey de Castro, F. 1995. Órdenes y desórdenes en la Selva Central: historia y economía de un espacio regional. Lima, Perú, IEP. 365 p.

Smith, RC. 1982. The dialectics of domination in Peru: Native communities and the myth of the vast Amazonian emptiness: an analysis of development planning in the Pichis Palcazu special project. s.l., Cultural Survival. 131 p.

Staver, C. 1989. Why farmers rotate fields in maize-cassava-plantain bush fallow agriculture in the wet Peruvian Amazon. Human Ecology 17(4): 401-426.

Staver, C; Simeone, R; Stocks, A. 1994. Land Resource Management and Forest Conservation in Central Amazonian Peru: Regional, Community, and Farm-Level Approaches among Native Peoples. Mountain Research and Development 14(2): 147-157.

Ulloa, A. 2004. La construcción del nativo ecológico: complejidades, paradojas y dilemas de la relación entre los movimientos indígenas y el ambientalismo en Colombia. Bogotá, Colombia, ICANH. 364 p.

Valadeau, C. 2016. De un convenio de recolección de muestras vegetales a la creación del Instituto de Etnobotánica Yánesha Domingo Huayul Espíritu: Estudio de caso en la selva central peruana. In Surrallés, A; Espinosa, O; Jabin, D. (eds.). Apus, caciques y presidentes. Estado y política indígena amazónica en los países andinos. Lima, Perú, IWGIA. p. 235251. 